\title{
Simulation of Phase Change Process of Nanofluid in Porous Media based on LBM
}

\author{
Shouguang Yao ${ }^{1, \mathrm{a}, *}$, Tao Huang ${ }^{1, \text { a }}$, Kai Zhao ${ }^{2, \mathrm{~b}}$, Jiangbang Zeng ${ }^{3, \mathrm{c}}$, \\ LuobinDuan ${ }^{1, \text { a }}$ \\ ${ }^{1}$ Jiangsu University of Science and Technology, Zhen Jiang, 212003, China; \\ ${ }^{2}$ Nantong CIMC tank equipment manufacturing, Nantong, 226002, China; \\ ${ }^{3}$ East China Jiaotong University, Nanchang, 330013, China; \\ azjyaosg@126.com, ${ }^{b} 335469407 @ q q . c o m,{ }^{c} 45957618 @ q q . c o m$ \\ * Corresponding Author: Shouguang Yao
}

\begin{abstract}
In this study, a two-dimensional partially filled porous medium structure model, which is able to simulate porous wick, was constructed via the stochastic collocation method. Different porosities were controlled, and water and Al2O3-water nanofluid were used as working media. In addition, a lattice Boltzmann model that can describe the liquid phase transition process in porous media was used to verify the combination of the nanofluid phase transition model and porous media structure. Bubble formation and growth, bubble fusion, and solid wall collision rebound phenomenon were observed through the simulation of the nanofluid boiling phase change process in the partly filled porous medium structure cavity. Moreover, the flow patterns of gas-liquid two-phase flow in nanofluid and pure liquid were compared. Results show that the phase change heat transfer capability of fluid is enhanced, the nanoparticle size increases, and the heat transfer ability of the fluid decreases with nanoparticle addition.
\end{abstract}

Keywords: porous media, nanofluid, gas-liquid phase transition, LBM

\section{Introduction}

With the development of power electronic device miniaturization and the increasing need for high-frequency, high-speed, high-power density, and high-rate electronic components per unit volume of heat power and heat flux density amplitude, the cooling requirements are also becoming high [1]. The application of new material technology in heat pipes is the direction for further development of heat pipe technology and improvement of the heat transfer capability of heat pipes. Nanometer-level metal or nonmetal oxide particle is added to the fluid, and the new heat transfer medium is obtained. The combination of good-quality heat conduction rate and heat pipe technology improves the heat transfer capacity of heat pipes $[2,3]$. Nanofluid with $\mathrm{Al}_{2} \mathrm{O}_{3}$ nanoparticles is suitable for heat transfer because of its large nanoparticle surface [4,5]. In addition, minimal movement strengthens the energy exchange between nanoparticle and liquid and subsequently strengthens the thermal conductivity of the work medium because of the brown force [6].

The boiling flow in porous media is widely observed in nature, chemical processes, environmental protection, electrical equipment, and some other fields [7]. Boiling heat transfer is widely used because of low temperature difference, high heat flow density, and other heat transfer characteristics, and the effect of porous wick heat pipe in the boiling heat transfer characteristics is increasingly explored [8,9]. The addition of porous wick in the tube can significantly enhance the boiling heat transfer of heat flow and reduce surface superheat. Amir F. Ali [10] applied a 
porous bed to tiny space cooling (integrated circuit) and adopted porous medium of different porosities (0.521 to 0.79$)$ and thicknesses $(80 \mu \mathrm{m}$ to $197 \mu \mathrm{m})$ into the cooling wall; the boiling heat transfer effect is threefold to sixfold of that in tablet. Porous structure and visual studies were conducted to obtain some evidence for the enhancement of boiling heat transfer in porous media [11,12]. Wang [13] used VOF (Volume of fluid) model coupling of porous media flow and heat transfer model of multiphase flow by adding a user-defined program. They achieved the simulation of pool boiling heat transfer in smooth and porous wall surface, thereby indicating that the results are consistent with the experimental results over a small heat range.

The phase transition of porous media is a typical cross-scale phenomenon, and the direct simulation of this kind of phenomenon is an important method to determine its mechanism by using the computational fluid dynamics method on pore scale. With the complexity of pore structure, the conventional method is difficult to use in solving the Navier-Stokes equation (finite volume, finite difference, and finite element methods) in pore-scale simulation of fluid solid boundary. Lattice Boltzmann method (LBM) is a kind of mesoscopic numerical method based on microscopic molecular dynamics; the complicated fluid solid boundary is easy to process, and the parallel efficiency is high, which is suitable for the parallel numerical simulation of the flow phase transition in porous media [14,15]. LBM uses the molecular dynamics model as foundation, and this method has the characteristics of microscopic and macroscopic methods. Therefore, LBM has a considerable advantage in dealing with complex flow problems that are multiscale and multiphysics. Nanofluid can enhance the single-phase convective heat transfer by using LBM [16-18]. Nevertheless, open published data to simulate the change process of nanofluid in two-phase heat transfer in porous media are not yet available.

The present study constructed a composite porous media wick structure of a twodimensional partially filled porous medium structure model via the stochastic collocation method, and the different porosities were controlled. Water and different concentrations of $\mathrm{Al}_{2} \mathrm{O}_{3}$-water nanofluid were selected by combining the phase transformation of the LB model proposed by Zhao et al. [19]. We also simulated the phase transformation of nanofluid in porous media and verified the combination of the nanofluid phase transition model with the structure of porous media. The formation, growth, and fusion of bubbles in porous structures were observed through the simulation of the phase transition of different nanofluid concentrations in porous media structure. This study also compared the nanofluid and pure liquid two-phase flow patterns. The fluid phase change heat capacity is strengthened, the nanometer particle size increases to a certain range, and the fluid transfer enhancement heat capacity is weakened with nanometer particle addition.

\section{Lattice Boltzmann Model of Phase Transition in Porous Media}

\subsection{Basic Multiphase Model of Nanofluid}

A theoretical model is applied to describe the gas-liquid phase change process; the evolution equations for this model can be expressed as follows [20]:

$$
f_{i}^{\sigma}\left(\boldsymbol{x}+\boldsymbol{e}_{i} \Delta t, t+\Delta t\right)-f_{i}^{\sigma}(\boldsymbol{x}, t)=\left(f_{i}^{\sigma e q}(\boldsymbol{x}, t)-f_{i}^{\sigma}(\boldsymbol{x}, t)\right) / \tau^{\sigma}
$$

where $\sigma=1$ represents liquid phase, $\sigma=2$ is gas phase, $i=0,1, \ldots, \mathrm{Q}$ represents the lattice velocity direction, $e_{i}$ is the lattice velocity vector, $x$ and $\Delta t$ are position vector and discrete time step, respectively. $\tau^{\sigma}$ is the dimensionless collision relaxation time of the $\sigma$ component of the fluid, $f_{i}^{\sigma}(x, t)$ is the population of the 
particles of $\sigma$ component with velocity $e_{i}$ at lattice $x$ and time t, and $f_{i}^{\sigma e q}(x, t)$ is the equilibrium distribution function which is defined as follows:

$$
f_{i}^{\sigma e q}=\omega_{i} f^{\sigma}\left[1+3 \frac{\mathbf{e}_{i} \cdot \mathbf{u}_{i}^{\sigma e q}}{c^{2}}+4.5 \frac{\left(\mathbf{e}_{i} \cdot \mathbf{u}_{i}^{\sigma e q}\right)^{2}}{c^{4}}-1.5 \frac{\mathbf{u}_{i}^{\sigma e q} \cdot \mathbf{u}_{i}^{\sigma e q}}{c^{2}}\right]
$$

Where $w_{i}$ is the weight coefficient, $e_{i}$ is the discrete speed, $f^{\sigma}=\sum f_{i}^{\sigma}$ is density of the $\sigma$ th component,the $\sigma$ th density is $\rho_{\sigma}=m_{\sigma} f^{\sigma}, m_{\sigma}$ is the $\sigma$ th weight; the $\sigma$ th momentum is $\rho_{\sigma} \mathbf{u}_{\boldsymbol{\sigma}}=m_{\sigma} \sum_{i} f_{i}^{\sigma} \mathbf{e}_{i}$, and $\boldsymbol{u}_{\sigma}^{e q}$ is equilibrium speed.

\subsection{The Force of Nanoparticles on the Base Fluid}

The nanofluid is composed of nanoparticles and the base fluid. In this paper, it is assumed that the nanoparticles have no shape and size. For the nanofluid, only the reaction force of the base fluid is considered.

The $F_{\sigma}$ are sum of internal forces and external forces that are affected by the removal of viscous force and pressure in a fluid. To nanoparticles, $F_{\sigma}$ include gravity, inter phase drag, Brown force and Fan Dehua's gravitational force. For the base liquid, it is the reaction force of the nanoparticles, that is, the resistance of the phase and the Brown force.

phase resistance [21]:

$$
F_{D}=-6 \pi \mu r_{p} \Delta u
$$

where, $\mu$ is the viscosity coefficient of the liquid $r_{p}$ is the radius of the nanoparticle and $\Delta u$ is speed difference.

Brown force [22]:

$$
F_{B}=\zeta \sqrt{\frac{\pi S_{0}}{\Delta t}}
$$

Among them, $\zeta$ is a Gauss random number that mean is zero, the variance is 1 ; $\Delta t$ is the particle Gauss motion time step; $S_{0}$ is the spectral intensity function.

$$
S_{0}=\frac{216 k_{B} T}{\pi^{2} \rho d_{p}^{5}\left(\frac{\rho_{p}}{\rho}\right)^{2} C_{c}}
$$

which is directly related to the the diffusion coefficient, then Brown force can be directly expressed by the diffusion coefficient:

$$
F_{B}=\zeta \sqrt{\frac{1}{D} \frac{2 k_{B}^{2} T^{2}}{\Delta t}}
$$

Where $\mathrm{T}$ is the average temperature of the nanofluid; $k_{B}$ is Boltzmann constant. $\mathrm{D}$ is Diffusion coefficient of nanoparticles.

$$
D=\frac{k_{B} T C_{C}}{3 \pi \mu d_{p}}
$$

Where $C_{c}$ is the slip coefficient, it can be expressed as:

$$
C_{c}=1+\frac{2 \lambda}{d_{p}}\left(1.257+0.4 e^{-\left(\frac{1.1 d_{p}}{2 \lambda}\right)}\right)
$$

Where, $\lambda$ is the average free travel of the base fluid molecules. The resultant force of the base fluid particles are:

$$
F=-\left(F_{D}+F_{B}\right)
$$




\subsection{Nanometer Fluid Pseudo Potential Force}

In a false potential model, the influence of the interaction force between particles is reflected by changing the speed of the equilibrium state in the equilibrium distribution function:

$$
\rho_{\sigma} \boldsymbol{u}_{\sigma}^{e q}=\rho_{\sigma} \boldsymbol{u}^{\prime}+\tau_{\sigma} \boldsymbol{F}_{\sigma}
$$

where $\rho_{\sigma}=m_{\sigma} f^{\sigma}$ is the mass density, $m_{\sigma}$ is the molecular mass of the $\sigma$ th component; $\boldsymbol{F}_{\sigma}=\boldsymbol{F}_{1}+\boldsymbol{F}_{2}+\boldsymbol{F}_{3}$ stands for the total force of particles, $\boldsymbol{F}_{1}, \boldsymbol{F}_{2}$ and $\boldsymbol{F}_{3}$ are the interaction force of fluid particles, and the interaction force between fluids and solid walls and other external forces, respectively.

$$
\boldsymbol{u}_{\sigma}^{e q}=\boldsymbol{u}^{\prime}=\sum_{\sigma}\left(\rho_{\sigma} \boldsymbol{u}_{\sigma} / \tau^{\sigma}\right) / \sum_{\sigma} \rho_{\sigma} / \tau^{\sigma}
$$

where the momentum of the $\sigma$ th component is $\rho_{\sigma} u_{\sigma}=m_{\sigma} \sum f_{i}^{\sigma} e_{i}$, and $\boldsymbol{u}_{\sigma}$ is the macro velocity of $\sigma$ th component.

To simulate phase change, the potential force must be considered. For the pseudo potential model, the pseudo potential function is described as follows [12]:

$$
\mathbf{V}_{\sigma \bar{\sigma}}\left(\mathbf{x}, \mathbf{x}^{\prime}\right)=G_{\sigma \bar{\sigma}}\left(\mathbf{x}, \mathbf{x}^{\prime}\right) \phi_{\sigma}\left(f^{\sigma}(\mathbf{x})\right) \phi_{\sigma}\left(f^{\bar{\sigma}}\left(\mathbf{x}^{\prime}\right)\right)
$$

where $\phi_{\sigma}\left(f^{\sigma}(x)\right)$ and $\phi_{\sigma}\left(f^{\bar{\sigma}}\left(x^{\prime}\right)\right)$ are the effective densities at position $x$ for $\sigma$ th component and at $\boldsymbol{x}^{\prime}=\boldsymbol{x}+\boldsymbol{e}_{i}$ for $\bar{\sigma}$ th component, respectively.

If only the isotropic interaction between neighboring nodes is considered, then Green function can be expressed as:

$$
G_{\sigma \bar{\sigma}}\left(\boldsymbol{x}, \boldsymbol{x}^{\prime}\right)= \begin{cases}0 & \left|\boldsymbol{x}-\boldsymbol{x}^{\prime}\right|>\Delta x \\ G_{\sigma \bar{\sigma}} & \left|\boldsymbol{x}-\boldsymbol{x}^{\prime}\right| \leq \Delta x\end{cases}
$$

where $G_{\sigma \bar{\sigma}}\left(\boldsymbol{x}, \boldsymbol{x}^{\prime}\right)=G_{\overline{\sigma \sigma}}\left(\boldsymbol{x}^{\prime}, \boldsymbol{x}\right), \Delta \boldsymbol{x}$ is the lattice length, $G_{\sigma \bar{\sigma}}\left(\boldsymbol{x}, \boldsymbol{x}^{\prime}\right)$ is the interaction strength between $\sigma$ th and $\bar{\sigma}$ th components, and the particle interactions of $\sigma$ th component can be taken as:

$$
\boldsymbol{F}_{1}(\boldsymbol{x})=-\phi_{\sigma}\left(f^{\sigma}(\boldsymbol{x})\right) \sum_{x} \sum_{\sigma} G_{\sigma \bar{\sigma}}\left(\boldsymbol{x}, \boldsymbol{x}^{\prime}\right) \phi_{\sigma}\left(f^{\bar{\sigma}}\left(\boldsymbol{x}^{\prime}\right)\right)\left(\boldsymbol{x}-\boldsymbol{x}^{\prime}\right)
$$

When the fluid comes into contact with the solid phase, solid density remains the same and the force between fluids and solid walls at the fluid-solid interface is

$$
\boldsymbol{F}_{2}(\boldsymbol{x})=-\phi_{\sigma}\left(f^{\sigma}(\boldsymbol{x})\right) \sum_{x} \sum_{\sigma} G_{\sigma w}\left(\boldsymbol{x}, \boldsymbol{x}^{\prime}\right) \phi_{w}\left(f^{w}\left(\boldsymbol{x}^{\prime}\right)\right)\left(\boldsymbol{x}-\boldsymbol{x}^{\prime}\right)
$$

where $\phi_{w}\left(f^{w}\left(\boldsymbol{x}^{\prime}\right)\right)$ is the effective density of solid wall, and $x^{\prime}$ is a constant if it is on the solid surface, whereas the effective density is zero; Green function $G_{\sigma w}\left(\boldsymbol{x}, \boldsymbol{x}^{\prime}\right)$ is the same as in Equation (14), but is characterized by the interaction intensity between fluids and solid walls; meanwhile, it can be used to reflect the soakage of fluid by adjusting the $G_{o w}\left(\boldsymbol{x}, \boldsymbol{x}^{\prime}\right)$.

For the phase change process in porous media, not only should the interaction force of fluid particles be considered, but also the interaction force between fluids and solid walls should be introduced into the Boltzmann model. As such, force term $\boldsymbol{F}_{2}$ is added on the basis of multiphase model mentioned in Section 2.1. To achieve no-slip velocity boundary conditions, bounce-back format is applied for the collision of fluid and solid walls. The interaction force between fluids and solid walls can be express as follows [16]:

$$
\boldsymbol{F}_{\sigma}^{s}=-f^{\sigma} \sum_{i} G_{\sigma w} s\left(\boldsymbol{x}+\boldsymbol{e}_{i}\right) \boldsymbol{e}_{i}
$$

The value of Boolean variable is either 0 (fluid lattice) or 1 (solid lattice), and it can describe the soakage (negative) and nonwetting (positive) of solids. 
After the addition of nanoparticles in the base liquid, the nanoparticles are subjected to the force including gravity, buoyancy, resistance, Vauls Ley Fan De, Brown force,but when the basic liquid phase transition, the force including the phase resistance, Brown force, intermolecular repulsion. Then formula (10) should be updated to:

$$
\rho_{\sigma} \mathbf{u}_{\sigma}^{\mathrm{eq}}=\rho_{\sigma} \mathbf{u}^{\prime}+\tau_{\sigma} \mathbf{F}^{\sigma}
$$

The $F^{\sigma}$ is the vector sum of all forces acting on the $\sigma_{\text {th }}$ component of the unit lattice volume.

$\sigma=1, F^{\sigma}$ represent the base fluid, its force include: resistance, Brown force, intermolecular repulsion force, gravity.

$\sigma=2, F^{\sigma}$ represent the gas phase,its force include: phase resistance force and gravity.

\subsection{Phase Transformation Model}

This paper focuses on a simple ideal phase change process (other minor factors are ignored), and the basic assumptions are as follows: (1) Calculation region reaches the saturation state; (2) Phase change rate is isotropic and has the same value in every discrete velocity directions; (3)To simulate phase change, heat flux q is supplied into the system. Based on the above assumptions, a phase change term is introduced to simulate the phase change and two-phase LBM is used to prompt the bubble fusion and growth. Therefore, the basic phenomenon of boiling can be simulated, and the macroscopic mass transfer equation can be taken as [18]:

$$
\frac{\partial \rho_{b} V_{b}}{\partial t}=-\Theta_{b \rightarrow r} \rho_{b} V_{b}+\Theta_{r \rightarrow b} \rho_{r} V_{r}, \frac{\partial \rho_{r} V_{r}}{\partial t}=\Theta_{b \rightarrow r} \rho_{b} V_{b}-\Theta_{r \rightarrow b} \rho_{r} V_{r}
$$

where $b$ and $r$ are liquid and gas phases, respectively, $\rho_{b}$ and $\rho_{r}$ are the densities of liquid and gas phases, and $V_{b}$ and $V_{r}$ are volume fractions of liquid and gas phases, which satisfy $V_{b}+V_{r}=1 \cdot \Theta_{b \rightarrow r}, \Theta_{r \rightarrow b}$ are phase change rates from liquid phase to gas phase and from gas phase to liquid phase, respectively.

This paper considers only the gasification process; hence Equation (18) can be simplified as:

$$
\frac{\partial \rho_{b} V_{b}}{\partial t}=-\Theta_{b \rightarrow r} \rho_{b} V_{b}, \frac{\partial \rho_{r} V_{r}}{\partial t}=\Theta_{b \rightarrow r} \rho_{b} V_{b}
$$

The change of two-phase volume fraction of gas and liquid over time during vaporization is $\quad V_{b}=1-\left[\left(1-e^{-\Theta_{b \rightarrow r} t}\right) /\left(1+(\gamma-1) e^{-\Theta_{b \rightarrow r} t}\right)\right]$ $V_{r}=\left(1-e^{-\Theta_{b \rightarrow r} t}\right) /\left(1+(\gamma-1) e^{-\Theta_{b \rightarrow r} t}\right) \quad$ (20) denotes to density ratio of gas and liquid; the expression of mass of gas phase and liquid phase change over time is

$$
M_{b}=\rho_{b} V_{b}=M_{b 0} e^{-\Theta_{b \rightarrow r} t}, M_{r}=\rho_{r} V_{r}=M_{b 0}\left(1-e^{-\Theta_{b \rightarrow r} t}\right)
$$

where $M_{b 0}=\rho_{b 0} V_{b 0}$ stands for initial quality of liquid phase, $\rho_{b 0}$ and $V_{b 0}$ are initial density and volume fraction of liquid phase, respectively. The densities of liquid and vapor in vaporization, $\rho_{b}$ and $\rho_{r}$ are

$$
\rho_{b}=\rho_{b 0}\left(1+(\gamma-1) e^{-\Theta_{b \rightarrow r} t}\right) / \gamma, \rho_{r}=\rho_{b 0}\left(1+(\gamma-1) e^{-\Theta_{b \rightarrow r} t}\right)
$$

By introducing the mesoscopic phase change rate $\theta_{i, b \rightarrow r}, \theta_{i, b \rightarrow r}=\Theta_{b \rightarrow r}$ according to Assumption (2). The phase change rate under constant temperature and pressure is associated with latent heat of gasification and it can be expressed as follows: 


$$
\theta_{i, b \rightarrow r}=\Theta_{b \rightarrow r}=\frac{q}{h}
$$

where $h$ is the latent heat of evaporation, which is equal to 1 .

Mesoscopic phase change equation is obtained as [25]:

$$
f_{i}^{b}=f_{i}^{b 0}-\Theta_{b \rightarrow r} f_{i}^{b 0}, f_{i}^{r}=f_{i}^{r 0}+\Theta_{b \rightarrow r} f_{i}^{r 0}
$$

where $f_{i}^{b 0}$ and $f_{i}^{r 0}$ are previous step distribution functions of the $f_{i}^{b}$ and $f_{i}^{r}$.

\section{Simulation Results and Analysis}

\subsection{Method Validation}

First, we constructed a partially filled porous medium model to simulate the porous wick by MATLAB based on the stochastic collocation method and used it in the phase change lattice Boltzmann model program model language by $\mathrm{C}++$. As shown in Figure 1, the porosity is $0.3,0.4,0.5$, and 0.6 . A porosity of 0.6 is similar to the heat pipe internal structure and is the closest to the experimental use of porous wick. Therefore, we used 0.6 as the porosity for simulation in this study.

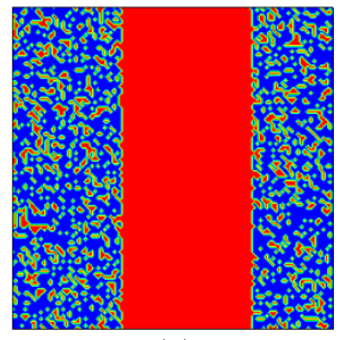

(a)

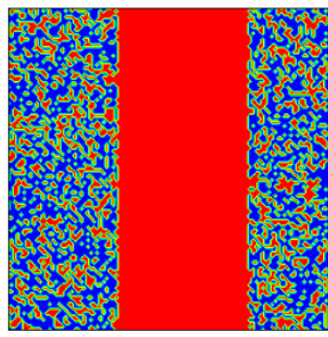

(b)

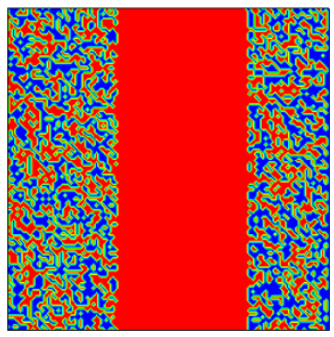

(c)

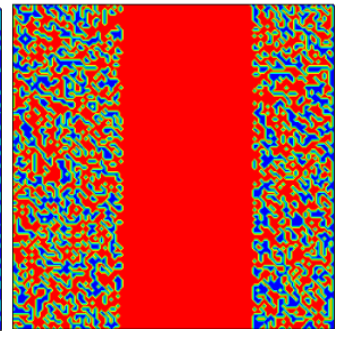

(d)

Figure 1. Pore Structure of Two-dimensional Porous Media with Different Porosities

We applied the phase change model to the simulation of phase transition in porous media. First, we assumed that the entire transition process is under isothermal conditions using the established phase change LB model with $200 \times 200$ mesh, and the porosity is 0.6 . The superheat of the system is 0.002 . At the initial time, the area is filled with liquid density of $\rho_{0}=8.0$. The relaxation time is $\tau=1.0$, the molecular weight is $M_{b}=M_{r}=1.0$, and the interaction strength of fluids is $G_{\sigma \bar{\sigma}}=0.3$. The interaction force between solid and liquid is $G_{\sigma w}=0.04$, and the gravity is $g=1 \times 10^{-5}$. All of the physical quantities are dimensionless. The periodic boundary scheme is used for the upper and lower boundaries, whereas the bounceback scheme is applied to the left and right boundaries. The interaction force of fluids and interaction force between fluids and walls are not considered. For the equation to evolve, the heat provides a disturbance of $1 \%$, with a computing iteration $t$ for a total of 1,000 steps. 

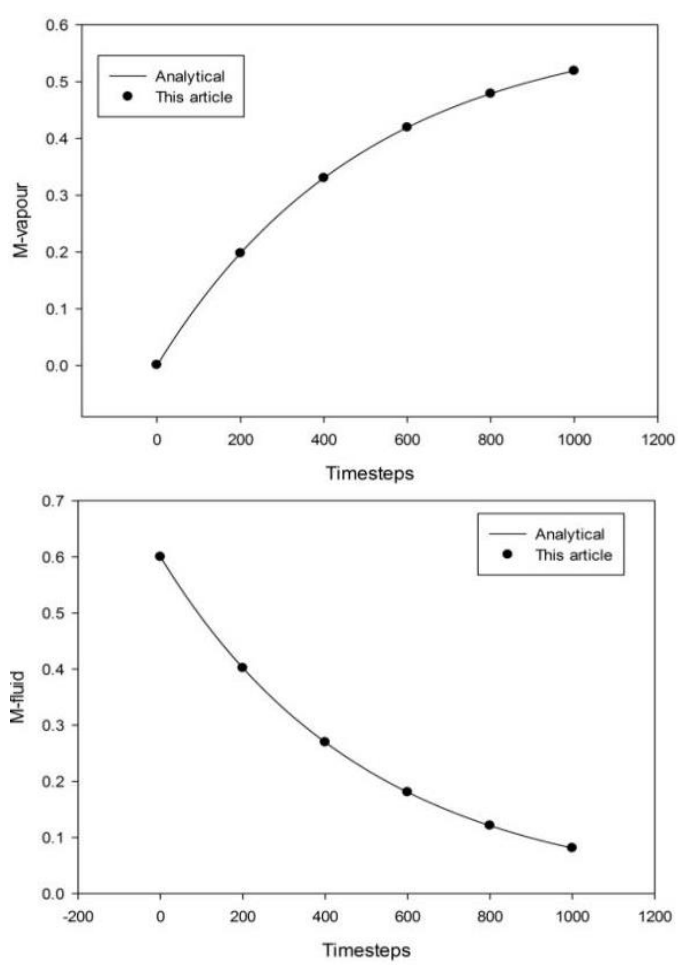

(a)

(b)

\section{Figure 2. Mass Change of the Gas (a) and Liquid (b) Phases with Time}

Figure 2 shows the change of the boiling process of gas and liquid phases in porous media with time. With the increase in time, the gas phase mass increases gradually and the quality of the liquid phase decreases gradually. This observation indicates that, during the transformation process, the gas phase gradually occupies the space of the liquid phase and eventually fills the porous medium-filled cavity.

\subsection{Comparison of Different Fluid Phase Transitions in Porous Medium}

Figures 3 to 7 present the two-phase diagram of pure liquids and different particle diameters of nanofluid filled in the porous medium model; the white part is the porous medium, the black part is the liquid phase, and the gray part is the gas phase, which is the bubble formation. The occurrence of phase transition in the porous medium is clearly observed. Initially, some small bubbles are formed gradually in the porous medium and blank area of the vicinity. The effect of fluid flow causes some bubbles to move toward the center region. Subsequently, the small bubbles grow. They undergo deformation, fusion, and rebound effectively in the face of the solid wall. With the increase in evolution time, the bubble diameter increases and the bubbles continue to collide with the surrounding air bubbles and aggregate. Massive bubbles are subsequently formed in the middle region. As the evolution time continues, the gas is in the continuous phase and the liquid is in the dispersed phase because of the continuous collision and polymerization of the massive bubbles in the cavity. Finally, the bubbles fill the entire region, including the porous medium and middle blank area, which reflects the infiltration characteristics of the bubbles and liquid. 


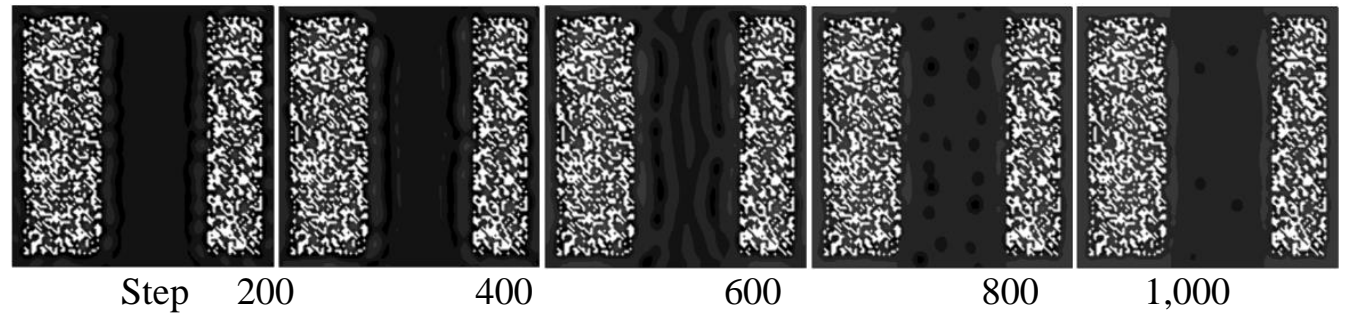

Figure 3. Two-phase Diagram of Pure Liquid in Partially Filled Porous Medium Model

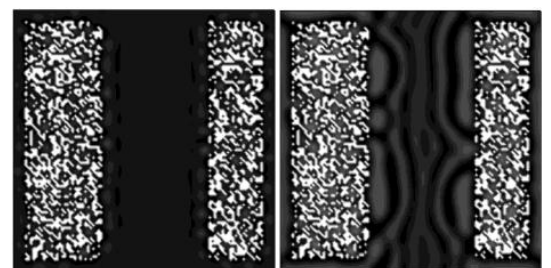

Step 200

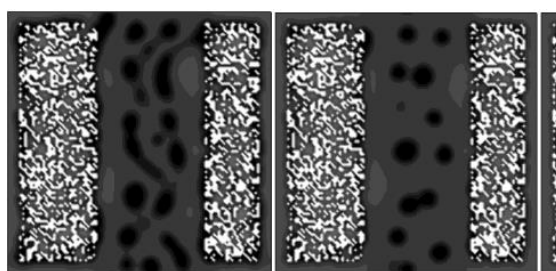

600

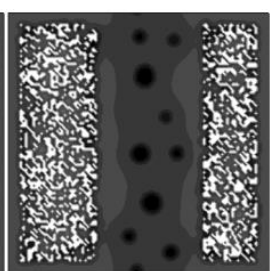

1,000

Figure 4. Two-phase Diagram of 20nm Nanoparticles in Partially Filled Porous Medium Model

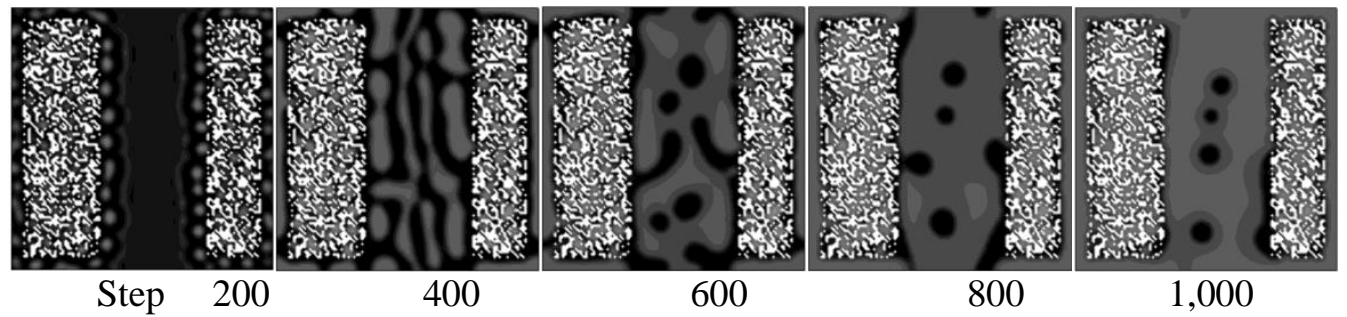

Figure 5. Two-phase Diagram of $15 \mathrm{~nm}$ Nanoparticles in Partially Filled Porous Medium Model

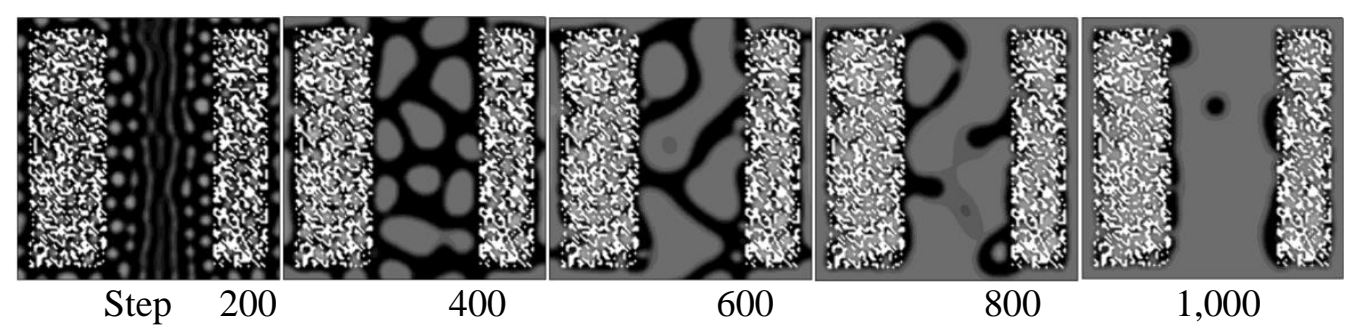

Figure 6. Two-phase Diagram of $10 \mathrm{~nm}$ Nanoparticles in Partially Filled Porous Medium Model

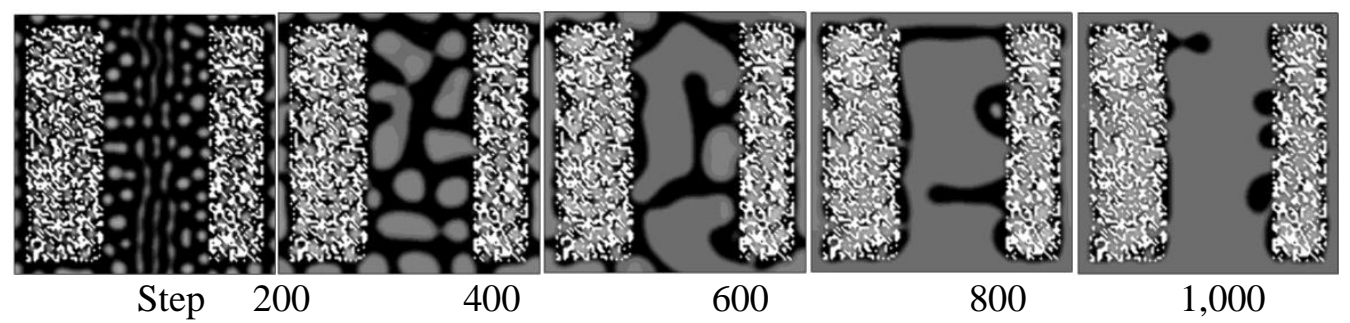

Figure 7. Two-phase Diagram of $5 \mathrm{~nm}$ Nanoparticles in Partially Filled Porous Medium Model 
Bubbles on the left and right wall surfaces and internal pore are formed and moved simultaneously. Part of the bubbles moves to the center of the region and aggregate in the center vertical channel. Moreover, the number of small bubbles and the void fraction in the entire region are both increased. Figures 4 to 7 show the gas-liquid two-phase flow patterns of pure liquid and nanofluids. The gas-liquid flow patterns of nanofluid and pure liquids are different. The bubble formation and fusion rates are also different, which is the same as that in the experiment in [18]. Such difference is attributed to the change of the thermal boundary layer near the heating surface of the moving state and the bubble layer structure caused by nanoparticle addition, which has a corresponding effect on the two-phase flow patterns. Furthermore, the heat transfer effect of nanofluid is stronger than that of pure liquid, and the heat transfer effect decreases gradually with the increase in the diameter of the nanoparticles. The added nanoparticles change the original liquid refrigerants, particularly increasing the heat capacity of the liquid, which improves the heating surface of bubble formation and growth. Consequently, the boiling heat transfer is enhanced, which is consistent with the result of Zhao Yanbing et al. [23]. The Brownian motion of nanoparticles with different diameters in the boiling process strengthens the heating surface in the vicinity of the thermal boundary layer disturbances. With the decrease in the particle diameter, the Brownian motion tends to be intense because of the heat transfer increase caused by particle migration, which strengthens the effect of heat transfer.
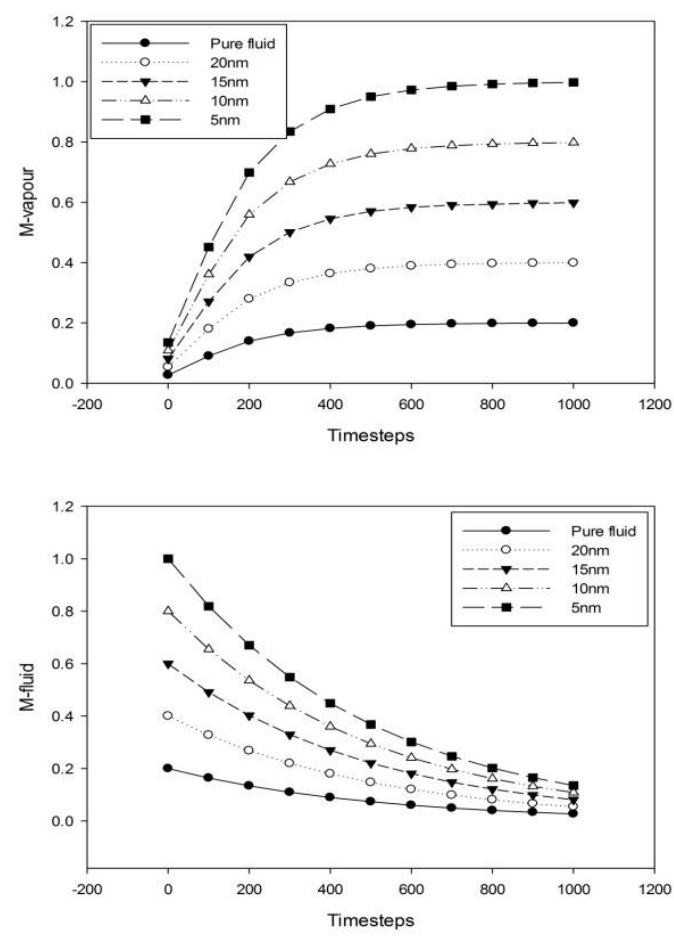

(a)

(b)

\section{Figure 8. Variation in the Mass of the Gas (a) and Liquid (b) Phases} with Different Particle Diameters

Figure 8 shows the gas (a) and liquid (b) phase quality changes of different particle diameters with time. At the same time step, the nanoparticle diameter decreases and the nanofluid vaporization rate increases gradually. Therefore, with the decrease in the nanoparticle diameter, the heat transfer effect of the nanofluid in the porous medium increases gradually. This change is attributed to the change of 
fluid property, particularly the improved heat capacity of the liquid, caused by nanoparticle addition. Thus, under the same conditions, the nanofluid phase changes first. As shown in the figure, at the same time step, the gas phase mass of the nanofluid is higher than that of the pure liquid (the liquid phase mass is less than the pure liquid). Under the diameter of the nanometer particles in this study, the Brownian movement tends to be intense with the decrease in the particle diameter. This observation also indicates that nanoparticle addition can enhance the heat transfer effect of the fluid.

\section{Conclusion}

The study on the phase change heat transfer in porous media is still in the primary stage, and the mechanism of heat transfer and influencing factors are still in the theoretical and experimental research. In this study, the LB model, which is suitable for the phase transition of porous media, is established. The method is based on the reaction force of the nanoparticles to the liquid phase.

(1) The porous medium model constructed by using MATLAB programming with the stochastic collocation method can effectively simulate porous wick void morphologies and reflect the randomness of wick and porous structure skeleton space arrangement.

(2) The nanofluid phase LB model was combined with the porous media, and the solid wetting and nonwetting properties were considered. Bubble formation, growth, merging, collision with solid wall, and rebound phenomenon were observed in the partially filled porous medium. The result verified the capability of the model to deal with the phase change of nanofluid in porous media.

(3) The boiling transfer enhancement capacity was stronger than that of the pure liquid with nanofluid as heat transfer medium. In the selected range, phase change can enhance the heat transfer capacity with the decrease in nanoparticle diameter.

(4) The mechanisms of heat transfer enhancement of nanoparticles were explained from a qualitative point of view. However, further study is needed to compare the experimental result. The model also supposes that the particles have no size or shape, which has certain deviation. Therefore, in future work, we will establish a nanofluid LB model conformed to porous wick heat pipe filled with porous media combined with particle dynamics.

\section{Acknowledgement}

This work was supported by National Natural Science Foundation of China under Grant NO.51176069.

\section{References}

[1] Janicki M, Napieralski A. Modelling electronic circuit radiation cooling using analytical thermal model [J].Microelectronics Journal, Vol. 31, No. 9, p. 781-785. (2000)

[2] Li Qingyou, Wang Wen, Zhou Genming. Study on the method of cooling of electronic components[J].Electronic devices. Vol.14, No, 4, p. 211-217. (2005)

[3] Chen Xiao, Zhang Renyuan, Mao Lingbo. Research and application of metal and oxide nano fluids [J]. Materials research and application. Vol. 2, No. 3, p. 173-176. (2008)

[4] Sarit K D, Nandy P,Wilfried R.Pool boiling characteristics of nano-fluids. [J]. Heat mass Transf. Vol. 46, No.4, p. 851-862. (2003)

[5] Kedzierski M A,Gong M. Effect of $\mathrm{CuO}$ nanolubricant on R134a pool boiling heat transfer.[J]. Vol. 5,No. 3, p. 791-799. (2009)

[6] Austin B. Gardner, Simon K. C. Lee, Elliot C. Woods, and Abhinav P. Acharya .BiomaterialsBased Modulation of the Immune System [J].BioMed Research International. Vol. 104, No. 13, p. 104-111. (2013)

[7] Ma H B, Wison C,Borgmeyer B. Effect of nanofluid on the heat transport capability in an oscillating heat pipe [J].Appl.Phys.Lett.Vol. 88, p.143116-143117. (2006) 
[8] Chein R, Huang G. Thermoelectric cooler applicatio electronic cooling [J]. Applied Thermal Engineering. Vol.24,(14):2207-2217. (2004)

[9] Li C H, Li T, Hodgins P,et al. Characteristics of pool boiling bubble dynamics in bead packed porous structures [J].Journal of Heat Transfer. Vol. 133, No. 3, p. 031-040. (2011)

[10] Ali A F,El-Genk M S. Spreaders for immersion nucleate boiling cooling of a computer chip with a central hot [J]. Energy Conversion and Management. Vol. 53, No. 1, p. 259-267. (2012)

[11] Wang Zheng, Peng Xiaofeng, Wang Buxuan. Experimental study on the characteristics of the boiling process of the core of the porous sphere $[\mathrm{J}]$. Journal of engineering and thermal physics. Vol. 23, No.1, p. 742-744. (2002)

[12] Wang Z,Peng X F, Ochterbeck J M. Dynammic bubble behavior during boiling in bead-packed structures[J].International Journal of Heat and Mass Transfer. Vol. 47, No. 22, p. 4771-4783. (2004)

[13] Wang Ji, Chen Wei, Mao Yubo. The characteristics of boiling heat transfer in a porous medium strengthened pool $[\mathrm{J}]$ Refrigeration technology, low temperature and superconductivity. Vol. 43, No. 3, p. 75-80. (2014)

[14] Guo zhao Li,Zheng chuguang. Theory and application of lattice Boltzmann method [M]. Beijing: Science Press. p. 202-204. (2009)

[15] Deng Y Q, Tang Z, Dong Y H. Lattice Boltzmann method for simulation propagating acoustic waves [J]. Chinese Journal of Computational Physics. Vol. 30, No. 6, p. 808-814. (2013)

[16] Guo Yali, Xu hehan, Shen Shengqiang Whelan. Rectangular cavity nanofluids Rayleigh Benard convection simulation with lattice Boltzmann method[J]. Journal of physics.Vol. 62, p. 144704 114711. (2013)

[17] Yang Y Z,Lai F H.Numerical study of flow and heat transfer characteristics of alumina-water nanofluids in a microchannel using the lattice Boltzmann method.[J] Heat mass Transf. Vol. 38, p. 607-614. (2011)

[18] Qi Cong, he Yurong, Yan Nan Sheng, Tian Lin Feng. Rectangular cavity nano natural convection in fluid two phase lattice Boltzmann simulation[J]. Journal of Engineering Thermophysics. Vol. 35, p. 282-286. (2014)

[19] Zhao Kai. Study on flow and heat transfer mechanism of porous media based on void scale [D] Nanjing University of Science and Technology, China, 2010. p. 77.

[20] Yao shouguang, Jia xinwang, Hu anjie, Li rongjuan. Analysis of nanofluids phase transition in pipe using the lattice Boltzmann method.[J]. International Journal of heat and Technology. Vol. 2, No. 33, p. 103-108. (2015)

[21] Xuan Yimin, Cui Tengfei, Li Qiang. Simulation of nano fluid enhanced mass transfer process based on lattice Boltzmann method[J]. Journal of chemical industry. Vol. 63, p. 41-46. (2012)

[22] Guo Zhaoli, Zheng Chuguang. The principle and application of lattice Boltzmann method [M]. Beijing: Science Press, (2009)

[23] Zhao Yanbing, Shi Ming Heng. Nano scale solid suspended particles enhanced pool boiling heat transfer experiment $[\mathrm{J}]$. Energy research and utilization of. Vol. 3, p. 18-23. (2001)

\section{Authors}

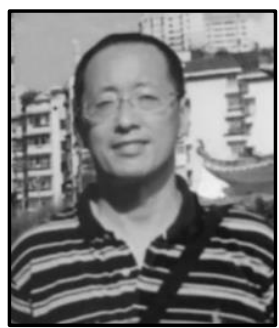

Shouguang Yao, Professor of School of energy and power engineering in Jiang su University of Science and Technology China, Jiang su. 
International Journal of Hybrid Information Technology

Vol. 10, No.2 (2017) 